\title{
2S-Soy Protein-Based Biopolymer as a Non-Covalent Surfactant and Its Effects on Electrical Conduction and Dielectric Relaxation of Polymer Nanocomposites
}

\author{
Zhuoyuan Zheng, Olaseeni Olayinka and Bin Li*
}

2S-soy protein, a biopolymer extracted from soy protein isolate (2S-SPI), was studied as a non-covalent surfactant for polymer nanocomposites. This study showed that 2S-SPI effectively improved carbon nanofibers (CNFs) dispersion in poly(vinylidene fluoride). 2S-SPI surfactant had remarkable impact on both electrical conduction and dielectric relaxation of the nanocomposites, particularly, at high temperatures. 2S-SPI modified CNFs caused coupling of conductivity relaxation and structural relaxations of the nanocomposites, in contrast to pristine CNFs. Both Maxwell-Wagner-Sillars and conductivity relaxations were enhanced at high temperatures by 2S-SPI, which made different contributions to electrical conduction of the nanocomposites with or without surface modification.

Keywords: 2S soy protein-based surfactant; Nanocomposites; Electrical and dielectric properties

Received 29 August 2018, Accepted 16 October 2018

DOI: $10.30919 / \mathrm{es} 8 \mathrm{~d} 766$

\section{Introduction}

Biomolecules are promising non-covalent surfactants, with low toxicity, good biodegradability, and environment friendliness. Meanwhile, the simplified processing/rinsing procedures of biomaterial functionalization require less time and energy consumption. Biomolecules, such as DNA, peptides and proteins have been studied as alternatives to traditional chemical surfactants. ${ }^{1-3}$ Ortiz-Acevedo et al. ${ }^{2}$ utilized reversible cyclic peptides (RCPs) to non-covalently functionalize and solubilize carbon single-walled nanotubes (SWNTs) in aqueous solution, through wrapping RCPs around the circumference of SWNTs and forming closed rings on SWNTs. Karajanagi et al. ${ }^{3}$ investigated a simple and scalable non-covalent functionalization procedure for solubilizing SWNTs in water using various commercial proteins intended for biomedical applications. Soy protein isolate (SPI), consisting of more than $90 \%$ protein, is a good protein provider in material engineering field. ${ }^{4} \mathrm{~J} \mathrm{Ji}$, et al. ${ }^{5}$ used SPI to treat CNTs and applied the protein-treated CNTs as fillers to prepare poly(ethylene oxide) (PEO) based nanocomposites. They discovered that SPI-modification of CNT was an effective and nondestructive method to enhance the CNT dispersion in polymer matrix. Their group ${ }^{1}$ further performed molecular dynamics (MD) simulations to demonstrate the effects of SPI as surfactants.

Soy protein is composed of several fractions, including 2S, 7S, and $11 \mathrm{~S}$, with molecular weights of approximately 18, 189 and 300 $\mathrm{kDa}$, respectively. ${ }^{6}$ 2S-SPI was reported to have applications as trypsin inhibitor in biomedical, ${ }^{7}$ biological, ${ }^{8}$ and food industry. ${ }^{9} 2 \mathrm{~S}$ SPI possesses high surface hydrophobicity and good chain flexibility,

Department of Mechanical Engineering Wichita State University1845 Fairmount St, Wichita, KS 67260-0133

*E-mail: bin.li@wichita.edu compared with high molecular weight protein fractions. ${ }^{10}$ Meanwhile, 2S-SPI contains numerous reactive groups including hydroxyls, amines, thiols, and carboxylic acids, and was reported to have tendency to interact with nanomaterials and adsorb onto their surfaces. ${ }^{6,11}$ Such advantages make it a good candidate for surface modification of nanomaterials. However, the applications of 2S-SPI as a surfactant for nanomaterials in polymer nanocomposites have not been studied yet.

Carbon nanomaterials have attracted intense attention in fabrication of polymer nanocomposites, due to their exceptional mechanical, ${ }^{12}$ electrical, ${ }^{13}$ and thermal $^{14}$ properties. However, the strong tendency of carbon nanomaterials to agglomerate hinders their practical applications. ${ }^{15}$ Various modification methods have been investigated to graft functional groups onto the surface of nanomaterials, thereby improving their chemical compatibility with solvents or polymers, leading to improved dispersion. ${ }^{16-18}$ Compared with covalent modification, non-covalent interactions are relatively moderate, and seldom cause damage to the nanomaterials. ${ }^{19}$ Various non-covalent surfactants have been studied, such as nonionic surfactants, such as polyoxyethylene 8 lauryl $\left(\mathrm{C}_{12} \mathrm{EO}_{8}\right),{ }^{17}$ nonylphenol ethoxylate (Tergitol NP-7) $;^{18}$ anionic surfactants, such as sodium dodecyl sulfate (SDS); $;^{20,21}$ cationic surfactants, such as dodecyl trimethyl ammoniumbromide (DTAB) $)^{22}$ and cetyltrimethylammounium 4-vinylbenzoate (CTVB), ${ }^{23}$ etc. Maestro et al. ${ }^{24}$ reported an experimental study on the improved wettability properties of nanoparticles through alkyltrimethylammonium modification, showing intricated electrostatic and hydrophobic interactions with nanoparticles. Osman et al. ${ }^{25}$ studied the nanocomposites of highdensity-polyethylene and dimethyldioctadecylammonium modified montmorillonite. The oxygen permeability coefficient of the nanocomposites was significantly reduced with surface modified montmorillonite, providing a strong barrier to oxygen and humidity, useful for food and drug packaging applications. SDS was found to 
be a good surfactant due to its ability to separate individual nanomaterials and stabilize nanomaterials in aqueous solutions. ${ }^{20,21}$ In addition, the strong influences of non-covalent surfactants on the mechanical properties, ${ }^{17}$ and thermal/fire stability, ${ }^{26}$ as well as electrical and thermal conductivity, ${ }^{27}$ etc., of the polymer nanocomposites have also been reported.

In this study, 2S-SPI surfactant, extracted from SPI, was utilized to modify carbon nanofibers (CNFs). Both untreated CNFs and 2SSPI treated CNFs (sCNFs) were compounded with poly (vinylidene fluoride) (PVDF) polymer to fabricate the nanocomposites. The roles of 2S-SPI as surfactants in electrical conduction and dielectric relaxation mechanisms of $\mathrm{PVDF} / \mathrm{CNF}$ nanocomposites were investigated. Particularly, the insufficiently understood effects of materials relaxation behaviors on electrical conduction of the nanocomposites were discussed in this study. At last, since high electrical conductivity of polymer nanocomposites would hide their natural structural relaxation behaviors, ${ }^{28,29}$ this study mainly focused on the nanocomposites before percolation of CNFs occurred.

\section{Materials and Methods}

\subsection{Materials}

Soy Protein Isolate (SPI, PRO-FAM 891) with high protein content $(>90 \%)$ was generously donated by Archer Daniels Midland (ADM). Carbon nanofiber (CNF) (PR-24-XT-HHT) was purchased from Pyrograf Products Inc. PVDF polymer with a weight average molecular weight (MW) of $534,000 \mathrm{~g} / \mathrm{mol}$ was purchased from Sigma-Aldrich.

\subsection{Sample Preparation}

Compared with high molecular weight fractions of SPI, $2 \mathrm{~S}$ fraction has a better solubility in Dimethyl sulfoxide (DMSO), which is principle for the extraction of $2 \mathrm{~S}$ fraction from SPI. To begin with, SPI was mildly denatured in DMSO solvent at $60{ }^{\circ} \mathrm{C}$ for 4 hours under magnetic stirring, producing a suspension containing both dissolved $2 \mathrm{~S}$ protein and undissolved solid. The suspension was filtered to remove all solid phases, and a clear 2S-SPI solution was collected, and confirmed by SDS-PAGE analysis. The detailed extraction procedure and analysis results were reported in our previous study. ${ }^{30}$

Afterwards, CNFs were added to as-prepared 2S-SPI solution with the CNF/2S-SPI weight ratio of $1: 1$ and 1:3, respectively. The mixtures were then sonicated (Branson 450) for $30 \mathrm{~min}$ with the power amplitude of $20 \%$. This procedure allowed the surface modification of 2S-SPI on CNFs. CNF solution without 2S-SPI modification was also prepared as a reference. The preparation for the PVDF/CNF and PVDF/sCNF (2S-SPI-treated CNFs) nanocomposites films started by completely dissolving weighted PVDF polymer into the CNF and sCNF solutions via mechanical mixing for 4 hours at 60 ${ }^{\circ} \mathrm{C}$. Solution-casting method was then applied to fabricate the films with a thickness of $\sim 55 \mu \mathrm{m}$. The obtained nanocomposites films were named according to the concentration of CNFs, and the modification conditions of CNFs, for example, $0.1 \mathrm{wt} \% \mathrm{CNF}$ and $1 \mathrm{wt} \% \mathrm{sCNF}-$ $1: 3$.

\subsection{Characterizations}

Morphologies of fracture surfaces of the nanocomposites were analyzed by Scanning Electron Microscope (SEM, FEI Versa 3D Dual Beam). Before SEM observation, the sample films were cryofractured in liquid nitrogen. Frequency dependent electrical conductivity and dielectric properties were analyzed using a HIOKI IM 3533-01 LCR meter. AC conductivity, dielectric constant and loss factor $\tan \delta$ were measured at $1.5 \mathrm{Vrms}$ in the frequency range of $1-$ $200,000 \mathrm{~Hz}$, and the temperature range of room temperature to $180^{\circ} \mathrm{C}$ with a temperature step of $20{ }^{\circ} \mathrm{C}$.

\section{Results and Discussion}

\subsection{Effects of 2S-SPI on Dispersion of CNFs}

Fig. 1 shows SEM images of the fracture surfaces of PVDF/1 wt $\%$ CNF nanocomposite with and without 2S-SPI modification. In $1 \mathrm{wt} \%$ CNF film Figs. 1a\&b, although high power sonication was applied to disperse CNFs, unsatisfactory dispersion of CNFs was still observed, with evident $\mathrm{CNF}$ aggregates (circled). The size of the aggregates was typically smaller than $10 \mu \mathrm{m}$. On the contrary, via 2S-SPI modification, sCNFs could be dispersed much more uniformly in PVDF matrix (Figs. 1c-f. The improved dispersion of sCNFs proved that 2S-SPI was a good surfactant for CNFs and enhanced their dispersion quality in PVDF. The increasing amount of 2S-SPI surfactant did not lead to noticeable differences in dispersion quality, comparing $1 \mathrm{wt} \% \mathrm{sCNF}-1: 1$ and $1 \mathrm{wt} \%$ sCNF-1:3 nanocomposites.
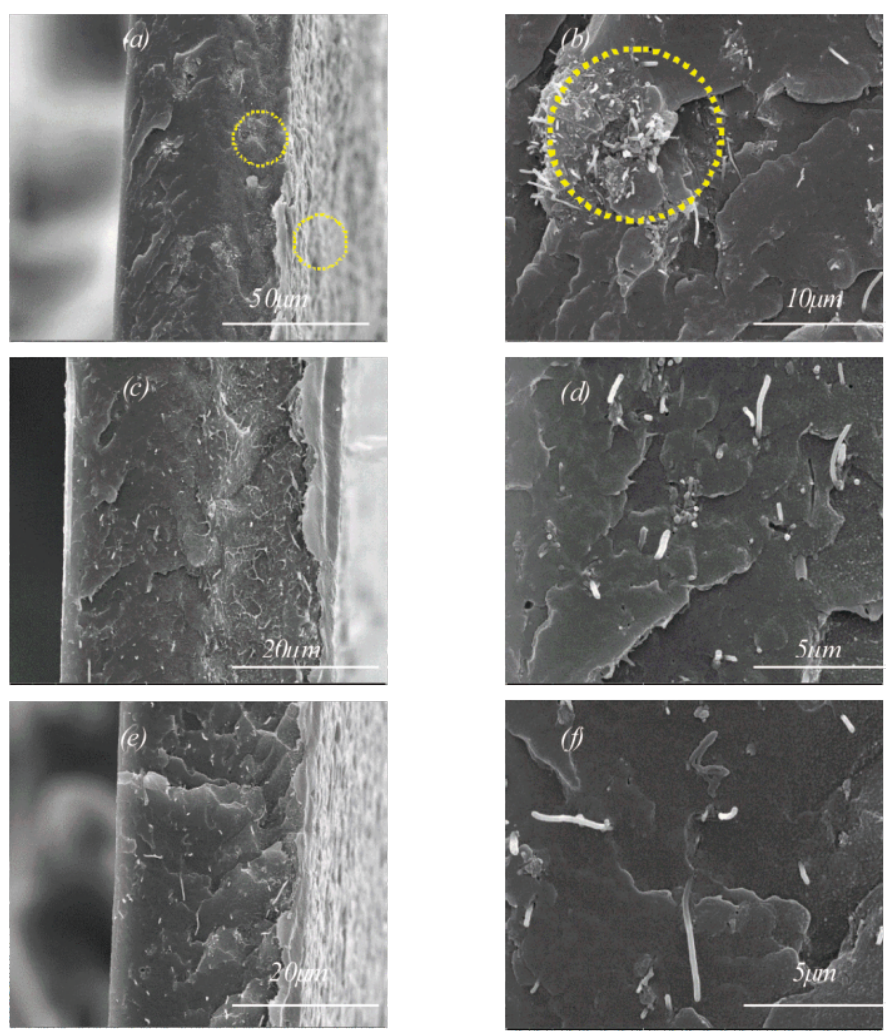

Fig. 1 SEM images of the fracture surfaces of $1 \mathrm{wt} \%$ nanocomposites: (a, b) 1 wt \% CNF; (c, d) 1 wt \% sCNF-1:1; (e, f) 1 wt \% sCNF-1:3.

\subsection{Electrical Conduction Analysis}

Fig. 2 shows the AC conductivity $\left(\sigma_{A C}\right)$ of pure PVDF and the nanocomposites at room temperature (RT). For pure PVDF and $\mathrm{PVDF} / \mathrm{CNF}$ nanocomposites containing $0.1 \mathrm{wt} \%$ and $1 \mathrm{wt} \% \mathrm{CNFs}$, $\sigma_{A C}$ showed an obvious frequency dependence in the whole frequency range, indicating their insulating nature. As for $2 \mathrm{wt} \% \mathrm{CNF}$ nanocomposite, the conductivity showed frequency independence, 

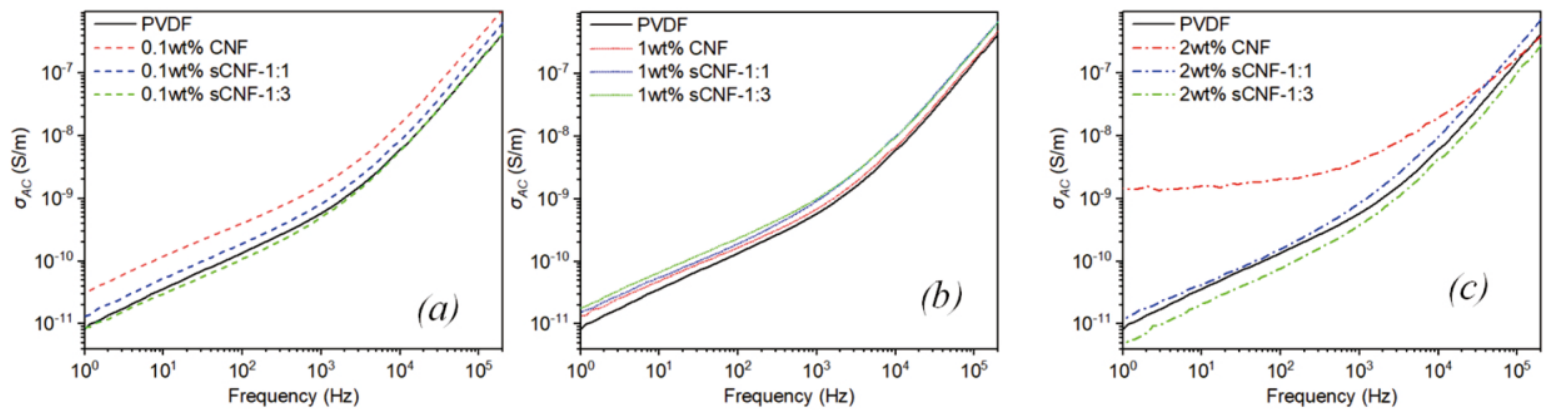

Fig. 2 AC Conductivity of pure PVDF and the nanocomposites at room temperature.

i.e. DC conductivity, as the frequency was lower than $100 \mathrm{~Hz}$. This suggested that percolation was happening around $2 \mathrm{wt} \% \mathrm{CNF}$ loading in PVDF/CNF nanocomposites. With 2S-SPI modification on CNFs, both $2 \mathrm{wt} \% \mathrm{sCNF}-1: 1$ and $2 \mathrm{wt} \%$ sCNF-1:3 nanocomposites exhibited frequency dependent $\sigma_{A C}$ in the whole frequency range, indicating the percolation moved to higher concentration level after the surface modification. Two reasons might be responsible for such changes. Firstly, 2S-SPI modified the surface properties of CNFs by forming an insulating coating layer, which hindered the mobility of charge carriers; Secondly, 2S-SPI did not only greatly improve the dispersion of CNFs, but also led to homogeneous distribution of CNFs. In previous studies, ${ }^{15,31,32}$ it was shown that homogeneous dispersion and distribution of conductive nanomaterials would delay the formation of conductive network.

High electrical conductivity of polymer nanocomposites would cover the true structural relaxation behaviors of materials, ${ }^{28,29}$ therefore, this study focused on the nanocomposites in pre-percolation region ( $\leq 1 \mathrm{wt} \% \mathrm{CNFs}$ ) to minimize the contributions of high electrical conductivity to the dielectric relaxation signals related with CNFs and 2S-SPI modification.

\subsubsection{Effects of 2S-SPI on Temperature Dependent Electrical Conductivity}

Fig. 3 and Fig. S1 show $\sigma_{A C}$ of pure PVDF and the nanocomposites. The frequency dependence of $\sigma_{A C}$ dramatically changed as temperature increased. Below $80{ }^{\circ} \mathrm{C}, \sigma_{A C}$ gradually increased with the increase of frequency, until the frequency exceeded approximately $10^{4} \mathrm{~Hz}$, when a sudden increase of the slope of $\sigma_{A C}$ curves was found in all PVDF and nanocomposites films. Such an increase was more dramatic at lower temperatures, leading to a reduction of $\sigma_{A C}$ as
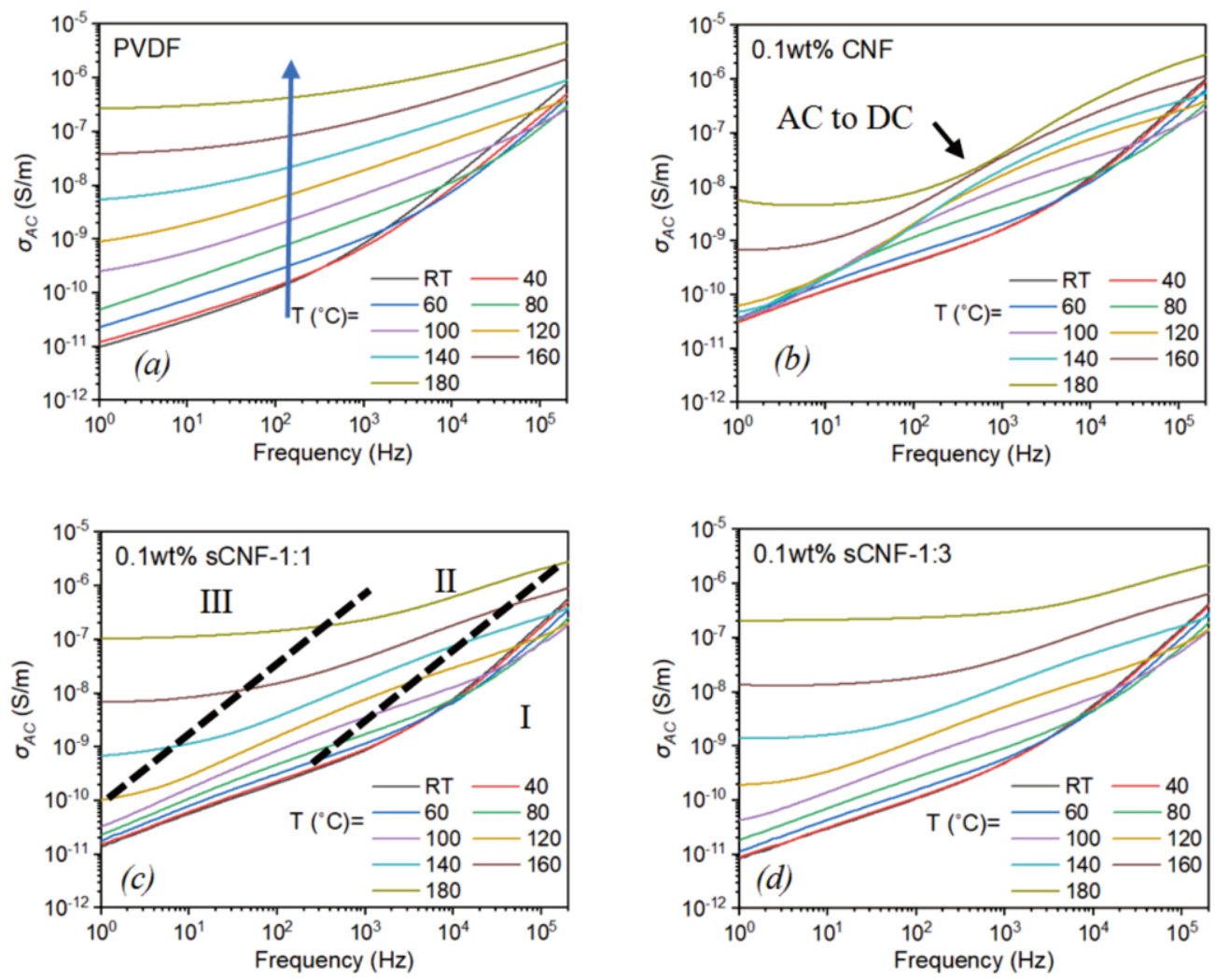

Fig. 3 AC conductivity of pure PVDF and $0.1 \mathrm{wt} \%$ nanocomposites from RT to $180{ }^{\circ} \mathrm{C}$ : (a) PVDF, (b) $0.1 \mathrm{wt} \% \mathrm{CNF}$, (c) $0.1 \mathrm{wt} \% \mathrm{sCNF}-1: 1$, (d) $0.1 \mathrm{wt} \% \mathrm{sCNF}-1: 3$. 
temperature increased from $\mathrm{RT}$ to $80{ }^{\circ} \mathrm{C}$ above $10^{4} \mathrm{~Hz}$. This phenomenon was believed to be related to the structural relaxations caused by localized hopping or re-orientational motion of dipoles in the glassy state, or segmental motions in amorphous structures of polymers. ${ }^{33-35}$ Similar phenomenon in PVDF composites was also reported in Ref. $36 .^{36}$ At temperatures above $100{ }^{\circ} \mathrm{C}$, frequencyindependent $\mathrm{DC}$ conduction was observed in low frequency range. The transition from AC to DC conduction suggested that the conduction mechanism switched from short-range to long-range hopping charge hopping in polymeric materials. ${ }^{37}$ The transition point from AC to DC conduction shifted towards higher frequency with increasing temperature.

Two transitions above divided the $\sigma_{A C}$ curves into three regions corresponding to three different conduction behaviors (Fig. 3c). Region I (low temperature and high frequency) was related to the structural relaxation behaviors around glass transition temperature or in amorphous structures; ${ }^{33,35}$ region II (medium temperature and frequency) was influenced by the accumulation of charge carriers at the interfaces between different phases; ${ }^{38,39}$ region III (high temperature and low frequency) indicated the long-range hopping of charge carriers.

Both CNFs and 2S-SPI surfactant showed remarkable impact on $\sigma_{A C}$, particularly, at high temperatures, regardless of the concentration of CNFs and sCNFs (Fig. 3 and Fig. S1). Untreated CNFs unexpectedly led to weakened electrical conductivity at low and medium frequencies (Fig. 3b), compared with pure PVDF polymer (Fig. 3a), which was recovered by $2 \mathrm{~S}$-SPI modification of CNFs
(Figs. 3c\&d, as discussed below.

To elucidate how CNFs and the 2S-SPI surfactant influenced the temperature dependent electrical conduction, the $\sigma_{A C}$ vs. $f$ curves were scaled to obtain master curves by normalization to DC conductivity and temperature (i.e. $\sigma_{D C}$ and $T$ ), ${ }^{40,41}$ as shown in Fig. 4 and Fig. S2. The three regions discussed above were more obvious in the master curves (Fig. 4a). For PVDF/CNF nanocomposites without 2S-SPI modification (Fig. 4b, Fig. S2a)), large deviation was observed in region I and region II. This deviation indicated a decoupling of the structural and conductivity relaxations. ${ }^{40}$ The main reason for such a decoupling was likely to be the movement of local dipoles and accumulated charges (interfacial polarization) at the interfaces between untreated CNFs and PVDF matrix. ${ }^{34}$ In the opposite, for 2S-SPI modified nanocomposites Figs. 4c\&d and Figs. S2b\&c, the scaled conductivity data well superimposed into a single master curve in all three regions, regardless of the amount of 2S-SPI surfactant, indicating that the coupling effect of conductivity and the structural relaxation mechanisms were barely interrupted by temperature. This indicated that 2S-SPI could effectively reduce the influences of interfacial polarization on electrical conduction. As far as we know, the influences of surfactants on the temperature dependence of electrical conductivity of nanocomposites have not been well understood yet.

Since $\sigma_{A C}$ of pure PVDF and the nanocomposites was influenced by multiple mechanisms, including structural relaxation, interfacial polarization, and short-range-to-long-range hopping conduction, labelled as region I, II, and III, respectively, the frequency dependent
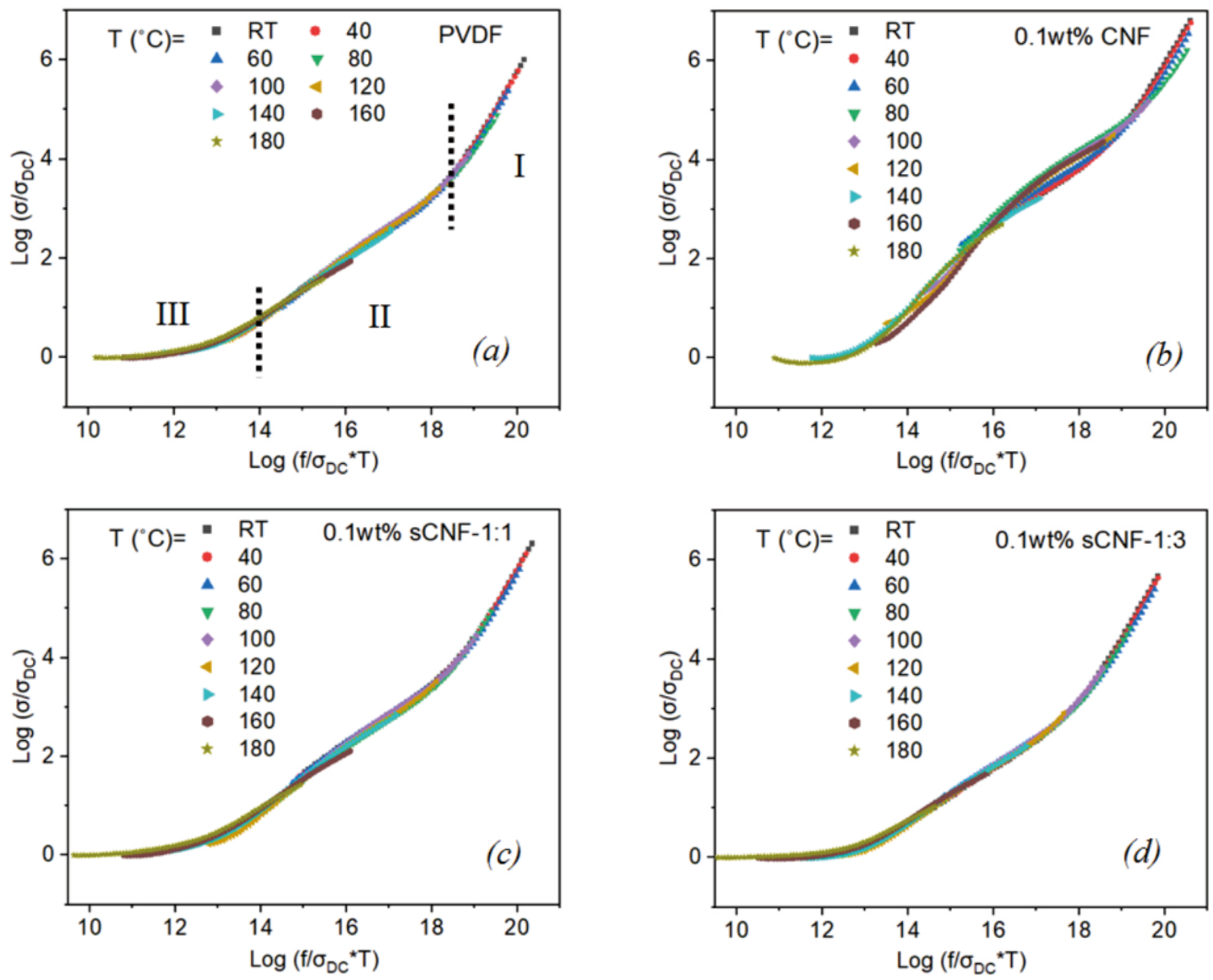

Fig. 4 Conductivity master curves for pure PVDF and $0.1 \mathrm{wt} \%$ nanocomposites. 

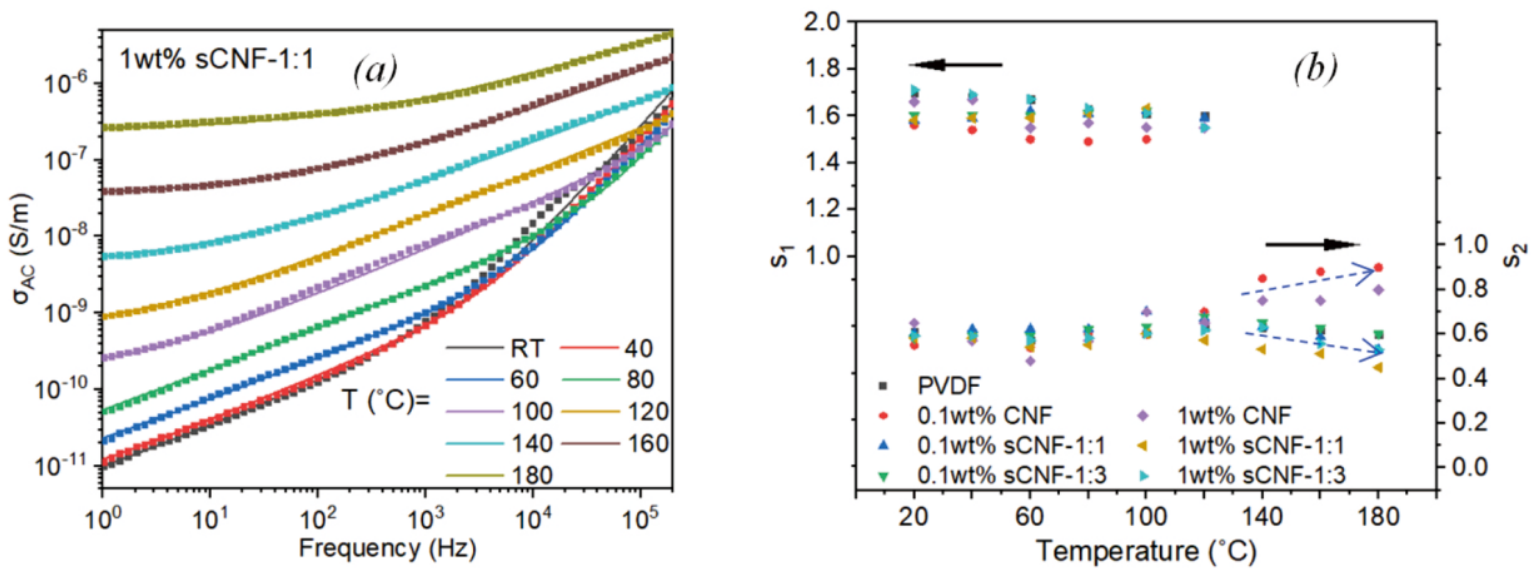

Fig. 5 (a) AC conductivity fitting example (1 wt $\%$ sCNF-1:1). Symbols: experimental data; solid lines: fitting results using equation (1); (b) universal exponents factor $s_{1}$ and $s_{2}$ as a function of temperature

$\sigma_{A C}$ could be described by a two-power-term universal law by Eq. $(1)::^{33,34}$

$$
\sigma_{A C}(f)=\sigma_{D C}+A_{1} \cdot f^{s_{1}}+A_{2} \cdot f^{s_{2}}
$$

$\sigma_{D C}$ is the DC conductivity (region III), $A_{1}$ and $A_{2}$ are pre-exponential factors, $s_{1}$ and $s_{2}$ are universal exponents, where $1<s_{1}<2$, dominating frequency region $>\mathrm{ca} .10^{4} \mathrm{~Hz}, s_{1}$ reflects the conductivity transition between region I and region II; $0<s_{2}<1$, dominating frequency region $<$ ca. $10^{4} \mathrm{~Hz}, s_{2}$ is related to the transition between region II and region III. ${ }^{34}$ An example of the fitted results of $1 \mathrm{wt} \%$ sCNF-1:1 nanocomposite, compared with experimental data, is shown in Fig. 5a. At temperatures above $120{ }^{\circ} \mathrm{C}$, the pre-exponential factor for high frequency region $A_{l}$ was fitted as 0 in all films, indicating that the transition between region I and region II did not take place within the testing range. As a result, when temperature was higher than $120{ }^{\circ} \mathrm{C}$, equation (1) decayed into the single-powerterm universal law: $\sigma_{A C}(f)=\sigma_{D C}+A_{2} \cdot f^{s_{2}}$. Therefore, the discussion on $s_{1}$ will only focus on the temperatures below $120{ }^{\circ} \mathrm{C}$.

The values of $s_{1}$ and $s_{2}$ as a function of temperature are presented in Fig. 5b. Smaller $s$ value indicates that the transition of the conductivity will be more shaped by the mechanisms located at lower frequencies. ${ }^{34} s_{1}$ values of PVDF/CNF nanocomposites were smaller than those of PVDF/sCNF nanocomposites at temperatures lower than $120{ }^{\circ} \mathrm{C}$, suggesting that, during the transition between region I and II, the interfacial polarization (region II, at lower frequencies than region I) had stronger influences on $\sigma_{A C}$ of $\mathrm{PVDF} / \mathrm{CNF}$ nanocomposites than $\sigma_{A C}$ of $\mathrm{PVDF} / \mathrm{sCNF}$ nanocomposites. As for $s_{2}$, at temperatures lower than $120{ }^{\circ} \mathrm{C}, s_{2}$ did not change much; however, when temperature elevated to $120{ }^{\circ} \mathrm{C}$, divergence of $S_{2}$ occurred: $s_{2}$ values of both $\mathrm{PVDF} / \mathrm{CNF}$ nanocomposites were much larger than those of pure $\mathrm{PVDF}$ and $\mathrm{PVDF} / \mathrm{sCNF}$ nanocomposites and kept increasing with the increase of temperature. The larger $s_{2}$ values of $\mathrm{PVDF} / \mathrm{CNF}$ films indicated that, during this transition (region II to III), the overall conduction behaviors were more shaped by the higher frequency mechanism, i.e. the interfacial polarization (region II). In one word, both transitions in $\mathrm{PVDF} / \mathrm{CNF}$ nanocomposites strongly depended on interfacial polarization. In contrast, the $s_{2}$ values of both pure PVDF and PVDF/sCNFs nanocomposites were smaller and decreased as temperature elevating, which revealed the enhanced contribution of long-range charge hopping (region III), but reduced contribution of interfacial polarization (region II) to the conduction behaviors. ${ }^{28,42}$

In summary, the discussions on both scaling of conductivity (Fig. 4) and universal law (Fig. 5) helped explain the differences in electrical conduction between $\mathrm{PVDF} / \mathrm{CNF}$ nanocomposites and $\mathrm{PVDF} / \mathrm{sCNF}$ nanocomposites. In PVDF/CNF nanocomposites, the interfacial polarization, related to short-range charge movement at the interfaces, had pronounced effects on the electrical conduction at low and medium frequencies. Particularly, when compared with pure PVDF, the addition of conductive CNFs did not favor the long-range charge hopping. This suggests that the charge carriers, including those from PVDF polymer, were largely confined in the vicinity of CNFs aggregates. On the other hand, in $\mathrm{PVDF} / \mathrm{SCNF}$ nanocomposites, the long-range charge hopping became the dominating factor in electrical conduction, replacing interfacial polarization. Although the insulating layer of 2S-SPI created high energy barriers for charge hopping, at high temperatures $\left(\geq 120^{\circ} \mathrm{C}\right)$, the thermally activated charge carriers gained high mobility and easily overcome the hopping energy barrier, even in sCNF-1:3 nanocomposites with higher amount of 2S-SPI surfactant. Meanwhile, more uniformly distributed sCNFs in the nanocomposites could largely minimize the mean geometric distances among sCNFs; ${ }^{43}$ thus, the long-range charge hopping occurred more easily in $\mathrm{PVDF} / \mathrm{sCNF}$ nanocomposites, which favored the electrical conduction in region III, in comparison with PVDF/CNF nanocomposites.

\subsubsection{Modification of Activation Energy of Electrical Conduction by 2S-SPI Surfactant}

Activation energy $\left(E_{a}\right)$ of $\mathrm{AC}$ conduction was investigated for all films. According to the Arrhenius law, the conductivity $\sigma_{A C}$ is dependent on temperature $T$, which is defined by Eq. (2): ${ }^{39,44}$

$$
\sigma_{A C}=\sigma_{0} \cdot \exp \left(-\frac{E_{a}}{R T}\right)
$$

where, $\sigma_{0}$ is a pre-exponential factor and represents the high temperature limit of conductivity, $E_{a}$ is the Arrhenius activation energy, $R=8.617 \times 10^{-5} \mathrm{eV} / \mathrm{K}$ is the universal gas constant, and $T$ is the absolute temperature. Fig. 6 shows $\sigma_{A C}$ as a function of the reciprocal of temperature $(1000 / T) .10 \mathrm{~Hz}$ and $10^{5} \mathrm{~Hz}$ were chosen here, since only one transition occurred at either very low $(\leq 10 \mathrm{~Hz})$ or very 

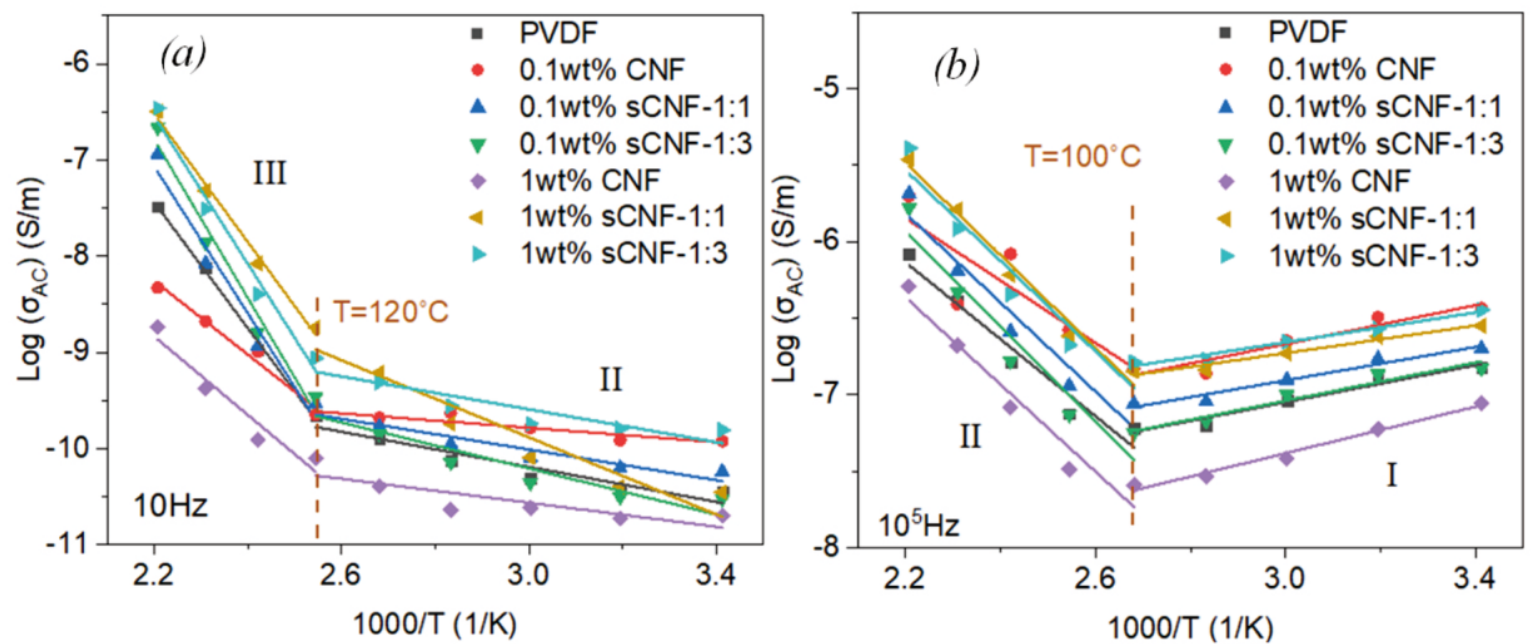

Fig. 6 Activation energy plots for pure PVDF and the nanocomposites at (a) $10 \mathrm{~Hz}$ and (b) $10^{5} \mathrm{~Hz}$.

high $\left(\geq 10^{5} \mathrm{~Hz}\right)$ frequency, as labeled in Fig. 3 and Fig. 6. $E_{a}$ curves over the entire temperature range were fitted into two separated straight lines for all PVDF/CNF and PVDF/sCNF nanocomposites, corresponding to the transition from region II to III at $10 \mathrm{~Hz}$, and from region I to II at $10^{5} \mathrm{~Hz}$, respectively. The transition temperatures were in accordance with previous analysis (Figs. $3 \& 4$ ), i.e. $\sim 120{ }^{\circ} \mathrm{C}$ for the transition between regions II and III at $10 \mathrm{~Hz}$, and $\sim 100^{\circ} \mathrm{C}$ for that between regions I and II at $10^{5} \mathrm{~Hz}$.

The calculated $E_{a}$ was given in Table 1 . At $10 \mathrm{~Hz}$, the addition of CNFs decreased $E_{a}$ for both regions II and III, compared with pure PVDF, while PVDF/sCNF nanocomposites possessed higher $E_{a}$ than $\mathrm{PVDF} / \mathrm{CNF}$ nanocomposites. The relatively lower $E_{a}$ of $\mathrm{PVDF} / \mathrm{CNF}$ nanocomposites in region II implied that the short-range movement of charge carriers in these nanocomposites was less restricted, and a large number of accumulated charges were possibly present at the interfaces between CNFs and PVDF. ${ }^{39}$ The lower $E_{a}$ in region III suggested lower energy barrier for the long-range charge hopping charges to show DC conduction in $\mathrm{PVDF} / \mathrm{CNF}$ nanocomposites. ${ }^{45}$ The low $\mathrm{E}_{\mathrm{a}}$ did not lead to high $\mathrm{DC}$ conduction in $\mathrm{PVDF} / \mathrm{CNF}$ nanocomposites, and this is probably because the charge carriers were mostly confined in the vicinity of CNF aggregates, besides the decoupling between conductivity relaxation and structural relaxations.

In $\mathrm{PVDF} / \mathrm{sCNF}$ nanocomposites, on the other hand, the increased $E_{a}$ in region II and III meant that, 2S-SPI surfactant, forming an insulating layer on the surface of $\mathrm{SCNFs}$, could enhance the energy barrier of charge hopping, but the increasing amount of 2S-SPI surfactant in sCNF-1:3 nanocomposites did not create much higher energy barriers. Meanwhile, it needs to be addressed here that high $E_{a}$ of PVDF/sCNF films in region III did not necessarily lead to low DC conductivity at high temperature $\left(\geq 120{ }^{\circ} \mathrm{C}\right.$, Fig. 3$)$, since the charge carriers were thermally activated to overcome the energy barrier caused by 2S-SPI modification. In this case, for long-range charging hopping, the dispersion quality was more crucial, as discussed above.

At $10^{5} \mathrm{~Hz}$, similar influences of CNF and sCNF were found at $\geq$ $100{ }^{\circ} \mathrm{C}$ (region II); however, in region I, negative $E_{a}$ was acquired. Negative $E_{a}$ indicated that, there were non-restricted free charge carriers, ${ }^{39}$ probably due to the structural relaxation at around glass transition temperature or in amorphous structures. ${ }^{35}$ The addition of CNFs or sCNFs did not significantly change the $E_{a}$ for region I.

Table 1. Activation energy (in $\mathrm{eV}$ ) of different conduction mechanisms at $10 \mathrm{~Hz}$ and $10^{5} \mathrm{~Hz}$.

\begin{tabular}{cccccccc}
\hline \multirow{2}{*}{$10 \mathrm{~Hz}$} & PVDF & $\begin{array}{c}0.1 \mathrm{wt} \% \\
\mathrm{CNF}\end{array}$ & $\begin{array}{c}0.1 \mathrm{wt} \% \\
\text { sCNF-1:1 }\end{array}$ & $\begin{array}{c}0.1 \mathrm{wt} \% \\
\text { sCNF-1:3 }\end{array}$ & $\begin{array}{c}1 \mathrm{wt} \% \\
\mathrm{CNF}\end{array}$ & $\begin{array}{c}1 \mathrm{wt} \% \\
\text { sCNF-1:1 }\end{array}$ & $\begin{array}{c}1 \mathrm{wt} \% \\
\text { sCNF-1:3 }\end{array}$ \\
\hline Region III & 0.56 & 0.33 & 0.66 & 0.71 & 0.35 & 0.57 & 0.66 \\
Region II & 0.079 & 0.032 & 0.069 & 0.103 & 0.054 & 0.173 & 0.074 \\
& & $0.1 \mathrm{wt} \%$ & $0.1 \mathrm{wt} \%$ & $0.1 \mathrm{wt} \%$ & $1 \mathrm{wt} \%$ & $1 \mathrm{wt} \%$ & $1 \mathrm{wt} \%$ \\
$10{ }^{5} \mathrm{~Hz}$ & PVDF & $\mathrm{CNF}$ & $\mathrm{sCNF}-1: 1$ & $\mathrm{sCNF}-1: 3$ & $\mathrm{CNF}$ & $\mathrm{sCNF}-1: 1$ & $\mathrm{sCNF}-1: 3$ \\
& & 0.18 & 0.25 & 0.27 & 0.25 & 0.26 & 0.25 \\
Region II & 0.22 & -0.055 & -0.047 & -0.054 & -0.055 & -0.050 & -0.048 \\
Region I & -0.052 & -0.054 & & & & \\
\hline
\end{tabular}




\subsection{Dielectric Relaxation Spectroscopy Analysis 3.3.1. Effects of 2S-SPI on Frequency Dependent Dielectric Properties}

Fig. S3 shows the dielectric properties of pure PVDF and all nanocomposites at room temperature and $180{ }^{\circ} \mathrm{C}$, respectively. At room temperature, the addition of either $\mathrm{CNFs}$ or $\mathrm{SCNFs}$ did not significantly affect the dielectric constant $\left(\varepsilon^{\prime}\right)$ or dielectric loss factor $\left(\tan \delta\right.$ ). At $180{ }^{\circ} \mathrm{C}$, all films, particularly, the $2 \mathrm{~S}$-SPI modified nanocomposites, acquired a rapid increase in $\varepsilon^{\prime}$ and $\tan \delta$ in low frequency region. In addition to the true dielectric polarization behaviors of materials, electrode polarization is also contributing to the low frequency dielectric properties during dielectric testing. ${ }^{46}$ The effect of electrode polarization often masks the relaxation behaviors of the materials at low frequencies and high temperatures. To overcome the electrode polarization effect and reveal the actual materials relaxations, electric modulus is frequently employed. ${ }^{28,38,46}$ The electric modulus $M^{*}$ is defined by the Eq. (3):

$$
M^{*}=\frac{1}{\varepsilon^{*}}, \quad M^{\prime}=\frac{\varepsilon^{\prime}}{\mathcal{E}^{\prime 2}+\varepsilon^{\prime 2}}, \quad M^{\prime \prime}=\frac{\varepsilon^{\prime \prime}}{\varepsilon^{\prime 2}+\varepsilon^{\prime \prime 2}}
$$

where, $M^{\prime}$ and $M^{\prime \prime}$ are the real and imaginary part of electric modulus.
In this study, instead of $\varepsilon^{\prime \prime}$ plots, $M$ " plots were used to analyze different relaxation behaviors affected by both CNFs and 2S-SPI surfactant.

\subsubsection{M" vs. Frequency Plots}

Fig. 7 and Fig. S4 illustrates the $M$ "' vs frequency plots from RT to $180{ }^{\circ} \mathrm{C}$. Dielectric relaxation behaviors of the nanocomposite films were sensitive to addition of CNFs and 2S-SPI modification. In general, some common phenomena were observed. At temperatures $<100{ }^{\circ} \mathrm{C}$, two major relaxation mechanisms were observed. The relaxation at $>$ ca. $10^{5} \mathrm{~Hz}$ (circled in red in Fig. 8) was related to the glass transition relaxation of PVDF and was denoted as $\alpha_{a}$ relaxation. ${ }^{47,48}$ The frequency and temperature ranges in this study were not able to provide sufficient information of $\alpha_{\mathrm{a}}$ relaxation, so that $\alpha_{a}$ relaxation was not the focus in this study. Meanwhile, the relaxation at frequencies $<10 \mathrm{~Hz}$ (circled in black in Fig. 8) involved two relaxation behaviors, i.e. $\alpha_{c}$ relaxation and interfacial polarization (also known as Maxwell-Wagner-Sillars (MWS) polarization), which were related to the relaxation dynamics of crystalline structures, and heterogeneity properties of the materials, respectively. The origin of $\alpha_{c}$ relaxation has several explanations, including the molecular movement of the polymer chains in the crystalline region due to orientation of the dipoles, ${ }^{49}$ or the motion of various imperfections: chain loops at the lamellar surface, chain
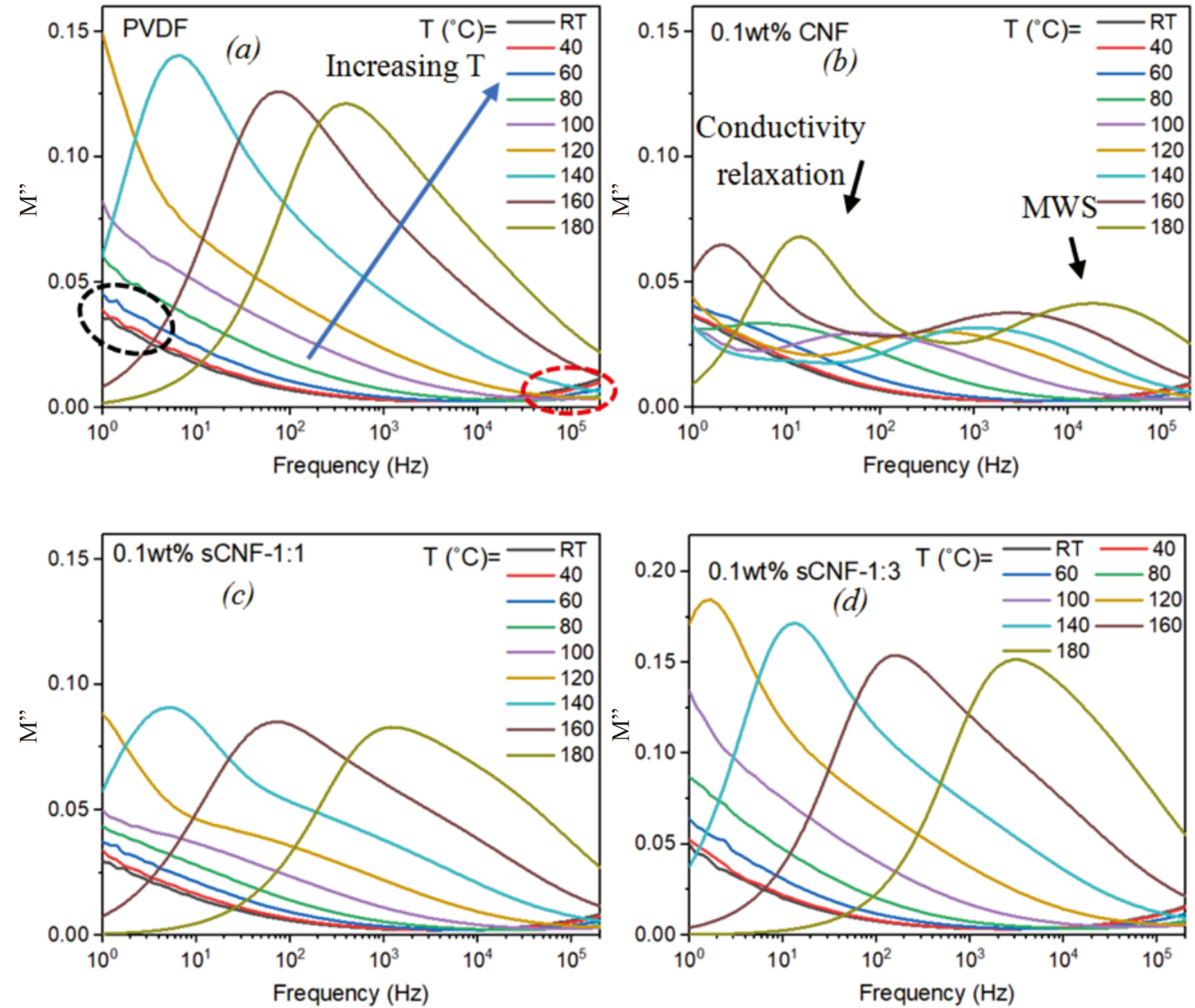

Fig. 7 M" vs. frequency plots of pure PVDF and $0.1 \mathrm{wt} \%$ nanocomposites from RT to $180{ }^{\circ} \mathrm{C}$. 
rotations and twisting within the interior of the crystals. ${ }^{50}$ MWS polarization usually occurs at the interface between different material structures, caused by separated charges accumulating at the internal phase boundaries. ${ }^{46,51}$ In this study, MWS relaxation came from two origins: interfaces between PVDF and CNFs, and interfaces between crystalline and amorphous PVDF. ${ }^{52}$

As temperature elevated, the $\alpha_{a}$ relaxation peak completely shifted out (towards higher frequency direction) of the measurement window; meanwhile, other relaxation peaks which were previously at low frequencies $(<10 \mathrm{~Hz})$ shifted to medium/high frequency (in blue arrow direction in Fig. 7). As temperature increased to around $120{ }^{\circ} \mathrm{C}$ (where DC conduction started to be observed in Figure 3), significant increases of $M$ " intensity occurred, and some complete relaxation peaks were observed in testing frequency range. In contrast to pure PVDF, in PVDF/CNF nanocomposites, two separated peaks were shown (Fig. 7 and Fig. S4), where the one at higher frequency was related to MWS relaxation, and the one at the lower frequency was conductivity relaxation. The conductivity relaxation symbolizes the transition between long-range and shortrange motions of charges along conductivity paths, ${ }^{53}$ often observed in PVDF at elevated temperatures. The obvious separation between conductivity relaxation and MWS relaxation observed on M" vs. Frequency plots was consistent with results from the scaled conductivity master curves in Fig.4, responsible for the highly deviated conductivity curves. Clearly, 2S-SPI modification was responsible for the merging of conductivity relaxation and MWS peaks in PVDF/sCNF nanocomposites (Fig. 7), i.e, coupling of these two relaxations. By comparing different sCNF nanocomposites (Figs.7b-c and Figs.S4b-c), it was found that the content of sCNFs and the amount of 2S-SPI surfactant had very slight effects on the coupling of these two relaxation mechanism. Moreover, PVDF polymer started to melt at around $140{ }^{\circ} \mathrm{C},{ }^{54}$ and $\alpha_{c}$ relaxation was absent at high temperatures. ${ }^{55}$

\subsubsection{M" vs. Temperature Plots}

M" vs. temperature plots at $10 \mathrm{~Hz}$ were also studied to better reveal and confirm different relaxation behaviors observed in Fig. 7. $\alpha_{c}$, conductivity, and MWS relaxations are clearly identified in Figure $8 .^{28}{ }^{50}$ Noticeably, $\alpha_{c}$ relaxation, which was not very clearly recognized in the M" vs. frequency figures (Fig. 7), was easily observed in Fig. 8. In pure PVDF, two broad peaks were shown at around $100{ }^{\circ} \mathrm{C}$ and $140{ }^{\circ} \mathrm{C}$, respectively. Based on the discussions on
Fig. 7, the peak at $100{ }^{\circ} \mathrm{C}$ was a combination of $\alpha_{c}$ and MWS relaxations, and the peak at $140{ }^{\circ} \mathrm{C}$ was related to conductivity relaxation. The addition of untreated CNFs, regardless of concentrations of CNFs, widely separated these three relaxation behaviors. The separation of $\alpha_{c}$ and MWS relaxations was obvious around $80{ }^{\circ} \mathrm{C}$ and $120{ }^{\circ} \mathrm{C}$. Meanwhile, the conductivity relaxation was not significant until high temperature was achieved $\left(>180{ }^{\circ} \mathrm{C}\right)$. After 2S-SPI modification, the PVDF/sCNF nanocomposites showed similar relaxation spectra with pure PVDF, with conductivity relaxations shifting back to much lower temperatures, suggesting the favorable effects of 2S-SPI modification on conductivity relaxation. The tendency of three relaxations merging together was also clear, due to the surface modification, in consistent with the results in Fig. 7.

In summary, according to the analyses on both M" vs. f (section 3.3.2) and M" vs. T (section 3.3.3) plots, the coupling of both conductivity relaxation and structural relaxation of the $\mathrm{sCNF}$ nanocomposites was observed, consistent with the findings in Fig. 4, regardless of the loadings of CNFs and the weight ratios between CNFs and 2S-SPI surfactant.

\subsubsection{Modification of Activation Energy of Dielectric Relaxations by $2 S-S P I$}

Self-convolution peak-fitting technique was applied to separate the combined relaxation peaks in Fig. 7, and investigate the contributions of CNFs and 2S-SPI to different relaxation mechanisms. An example of the peak fitting results was shown in Fig. S5. At temperatures $\leq 100{ }^{\circ} \mathrm{C}$, three relaxation peaks were separated, including MWS, $\alpha_{c}$ relaxation, and $\alpha_{a}$ relaxation; while at temperatures $>120{ }^{\circ} \mathrm{C}$, the relaxation peaks were divided into conductivity relaxation, MWS, and $\alpha_{c}$ relaxation, where $\alpha_{c}$ relaxation vanished when temperature $\geq 160{ }^{\circ} \mathrm{C}$, because of the melting of PVDF. ${ }^{55}$

$f$ for each relaxation peak at different temperatures was revealed according to peak fitting results. The thermally activated mechanisms of conductivity, MWS, and $\alpha_{c}$ relaxation processes were then studied by Arrhenius law Eq (4), and the activation energy $E_{a}$ was calculated by Eq. (4). ${ }^{35,53}$

$$
f=f_{0} \cdot \exp \left(-\frac{E_{a}}{R T}\right)
$$
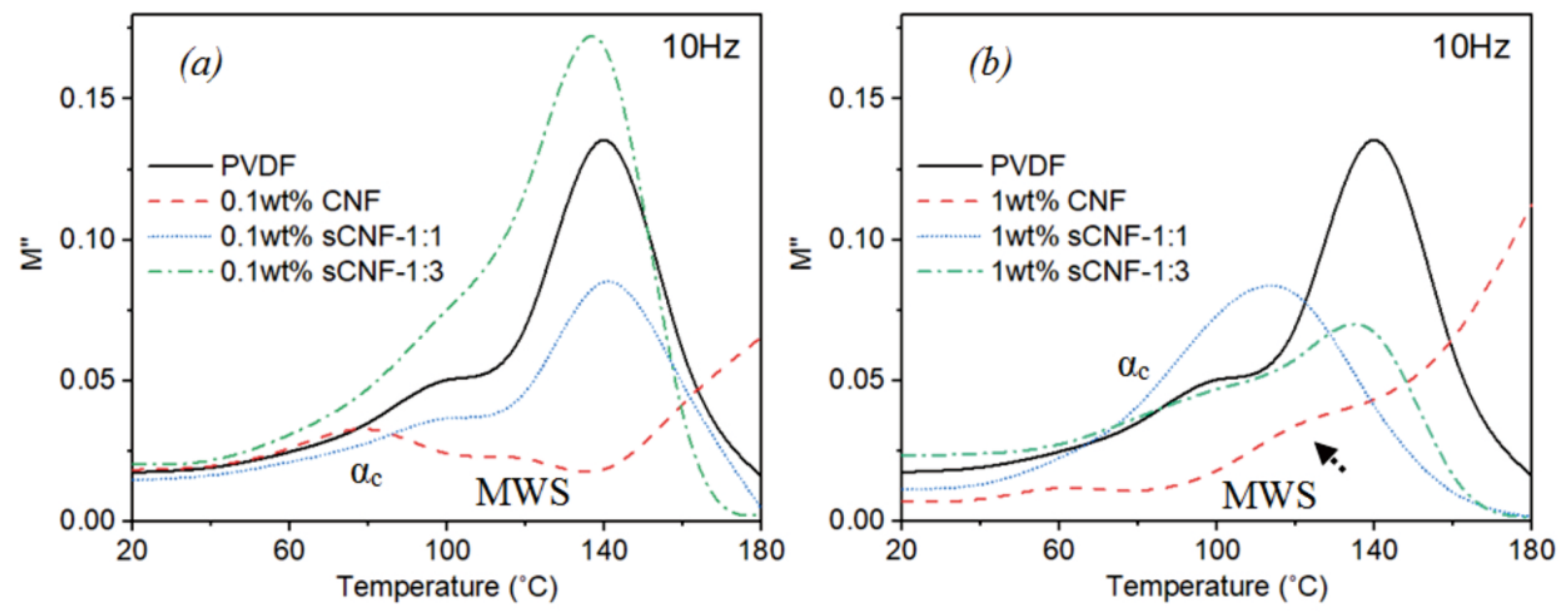

Fig. 8 M" vs. temperature plots of pure PVDF and the nanocomposites at $10 \mathrm{~Hz}$. 

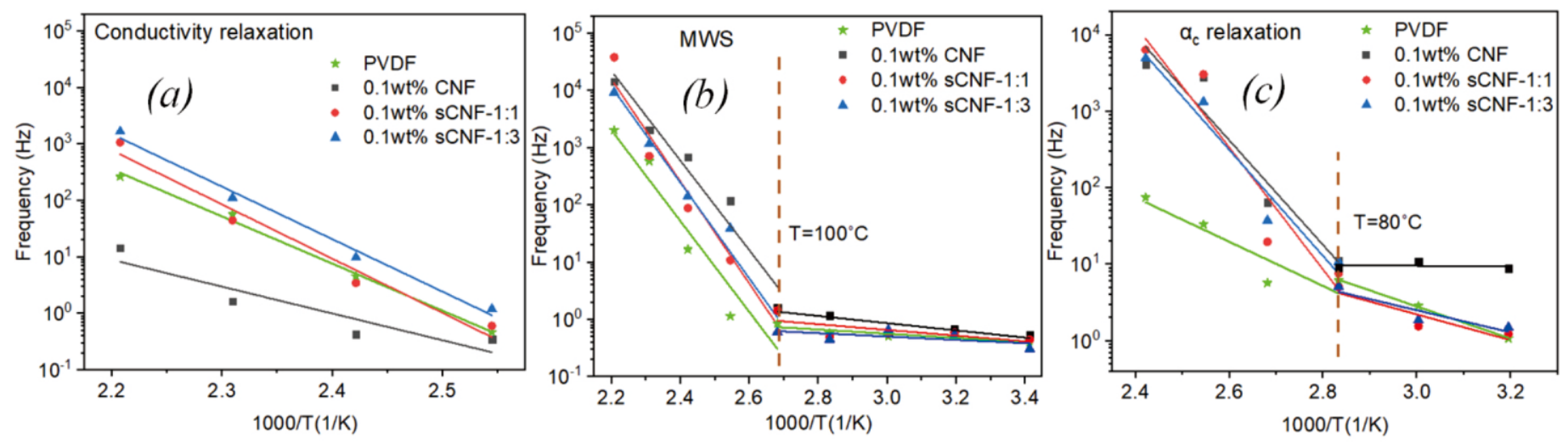

Fig. 9 Arrhenius plots for (a) conductivity relaxation, (b) MWS relaxation, and (c) $\alpha_{\mathrm{c}}$ relaxation for pure PVDF and $0.1 \mathrm{wt} \%$ nanocomposites.

where, $f$ is the peak frequency for each relaxation process at different temperatures, $f_{0}$ is the pre-exponential frequency, $E_{a}$ is the Arrhenius activation energy, $R=8.617 \times 10^{-5} \mathrm{eV} / \mathrm{K}$ is the universal gas constant, and $T$ is the absolute temperature.

Fig. 9 and Fig. S6 illustrate the Arrhenius behaviors of the three relaxation processes of $\mathrm{PVDF} / \mathrm{CNF}$ nanocomposites, indicating the relaxations were thermally activated process. ${ }^{29}$ According to the temperature dependence of each relaxation process (Fig. 8) and selfconvolution peak fitting results, the conductivity relaxation was investigated only at temperatures $\geq 120{ }^{\circ} \mathrm{C} ; \alpha_{\mathrm{c}}$ relaxation was only considered at $\leq 140{ }^{\circ} \mathrm{C}$; and MWS relaxation was investigated in the whole temperature range. Except conductivity relaxation (Fig. 9a, Fig. S6a), both MWS (Fig.9b, Fig. S6b)) and $\alpha_{c}$ relaxation (Fig. 9c, Fig. S6c)) showed two different temperature dependences. The calculated $E_{a}$ for three relaxations were listed in Table 2. Activation energy $E_{a}$ of conductivity relaxation implies the activation barrier of charge transport; $E_{a}$ of MWS relaxation originates from the accumulation of charge carriers blocked at the interfaces; ${ }^{53}$ and $E_{a}$ of $\alpha_{c}$ relaxation reflects the restraint of movement and re-orientation of dipoles and imperfections in crystalline phase. ${ }^{49}$
Compared with pure PVDF, addition of CNFs reduced the $E_{a}$ of both conductivity relaxation and MWS relaxation $\left(\geq 100{ }^{\circ} \mathrm{C}\right)$, suggesting that the restriction of both dipole motion caused by free charges and accumulated charges at interfaces became weak, ${ }^{56}$ consequently made the interfacial polarization and conductivity relaxation easy to occur. After 2 S-SPI modification, both $E_{a}$ values were largely promoted, suggesting increased temperature dependence by 2 S-SPI surfactant because of more restrictions on free charges and accumulated charges. $E_{a}$ of $\alpha_{c}$ relaxation of PVDF polymer above $80{ }^{\circ} \mathrm{C}$ was largely improved by adding CNFs, and further enhanced by 2S-SPI modification, indicating the energy barrier for the rotation of dipoles trapped by the crystal lattice was improved by the addition of CNF and SCNF. $E_{a}$ for MWS below 100 ${ }^{\circ} \mathrm{C}$ and $\alpha_{\mathrm{c}}$ relaxation below $80{ }^{\circ} \mathrm{C}$ were much lower than those at higher temperatures, and the effects of $\mathrm{CNF}$ and $\mathrm{sCNF}$ were less significant.

\subsubsection{Analysis of High Temperature Relaxations via Havriliak-Negami Model}

Table 2. Activation energy $E_{a}$ (in $\mathrm{eV}$ ) of conductivity relaxation, MWS and $\alpha_{\mathrm{c}}$ relaxation processes.

\begin{tabular}{|c|c|c|c|c|c|c|c|c|}
\hline & & & $0.1 \mathrm{wt} \%$ & $0.1 \mathrm{wt} \%$ & $0.1 \mathrm{wt} \%$ & $1 \mathrm{wt} \%$ & $1 \mathrm{wt} \%$ & $1 \mathrm{wt} \%$ \\
\hline & & 1 VDT & $\mathrm{CNF}$ & 1 & 3 & $\mathrm{CNF}$ & 1 & 3 \\
\hline \multicolumn{2}{|c|}{ relaxation } & 0.72 & 0.41 & 0.83 & 0.80 & 0.29 & 0.69 & 0.85 \\
\hline MWS & $\geq 100^{\circ} \mathrm{C}$ & 0.68 & 0.67 & 0.77 & 0.72 & 0.46 & 0.70 & 0.81 \\
\hline \multirow[t]{2}{*}{ relaxation } & $\leq 100^{\circ} \mathrm{C}$ & 0.032 & 0.054 & 0.043 & 0.025 & 0.035 & 0.023 & 0.040 \\
\hline & $\geq 80{ }^{\circ} \mathrm{C}$ & 0.25 & 0.59 & 0.69 & 0.60 & 0.43 & 0.66 & 0.72 \\
\hline relaxation & $\leq 80{ }^{\circ} \mathrm{C}$ & 0.181 & 0.127 & 0.131 & 0.135 & 0.005 & 0.145 & 0.125 \\
\hline
\end{tabular}


According to the discussion on M" plots in Sections 3.3.2 and 3.3.3, 2S-SPI had more significant influences on conductivity and MWS relaxations, particularly at high temperatures, which further affected electrical conduction of the nanocomposites. Meanwhile, both conductivity relaxation and MWS relaxation mostly occurred within out testing range at temperature $>140{ }^{\circ} \mathrm{C}$, (Fig. 7). Therefore, the discussion will primarily focus on conductivity and MWS relaxation mechanisms at $180^{\circ} \mathrm{C}$ and $160{ }^{\circ} \mathrm{C}$. Havriliak-Negami (HN) model function was applied, combined with the conductivity term (reflecting contributions of DC loss to dielectric relaxation), as Eq. $(5)::^{29,57}$

$$
\varepsilon^{\prime}=\varepsilon^{\prime}-i \varepsilon^{\prime \prime}=-i\left(\frac{\sigma_{d c}}{\varepsilon_{0} \omega}\right)^{N}+\varepsilon_{\infty}+\sum_{k=1,2}\left[\frac{\Delta \varepsilon_{k}}{\left(1+\left(i \omega \tau_{H N k}\right)^{\alpha_{k}}\right)^{\beta_{k}}}\right]
$$

where, $\sigma_{D C}$ is the DC conductivity, $\omega=2 \pi f$ is the angular frequency, $\varepsilon_{0}=8.854 \times 10^{-12} \mathrm{~F} / \mathrm{m}$ is the vacuum permittivity; the value of exponent $N$ is between 0 and 1 , in this study, the fitted $N$ values were every close to 1 for both pure PVDF and the nanocomposites, when temperature is above $100{ }^{\circ} \mathrm{C} ; \varepsilon_{\infty}$ is the limiting high-frequency permittivity, $\Delta \varepsilon_{k}$ denotes the relaxation strength of the $\mathrm{k}^{\text {th }}$ relaxation process, $\tau_{H N ~}{ }_{k}$ is the HM relaxation time, and $\alpha_{k}$ and $\beta_{k}$ are shape parameters related to symmetric and asymmetric broadening of the relaxation peaks, respectively.

Fig. 10 presents both fitted results and measured data of pure PVDF and 1 wt $\%$ sCNF-1:1 nanocomposite at $180{ }^{\circ} \mathrm{C}$, as an example, proving good fitting results of $\mathrm{HN}$ model. Dielectric relaxation time obtained from $\mathrm{HN}$ fitting, $\tau_{H N k}$, was also used to calculate the activation energy for each relaxation process using Eq. (4), and the results (Table S1) showed similar variation trends to those calculated through $M^{\prime \prime}$ fitting (Table 2). The fitting factors, including shape parameters $\alpha_{k}$ and $\beta_{k}$, relaxation time $\tau_{H N k}$ and dielectric relaxation strength $\Delta \varepsilon_{k}$ for $k$ relaxation process at $180{ }^{\circ} \mathrm{C}$ and $160{ }^{\circ} \mathrm{C}$ were listed in Table 3 and Table S2, respectively. Both $\alpha_{k}$ and $\beta_{k}$ did not show clear dependence on the CNFs and 2S-SPI modification. Three types of relaxations were typically observed: Debye relaxation $(\alpha=1 ; \beta=1)$; Cole-Cole relaxation $(\alpha \neq 1 ; \beta=1)$ or Cole-Davidson relaxation $(\alpha=1 ; \beta \neq 1)$. This suggests the complex synergetic influences of CNFs and 2S-SPI surfactant on dielectric relaxation in the nanocomposites, causing different symmetric or

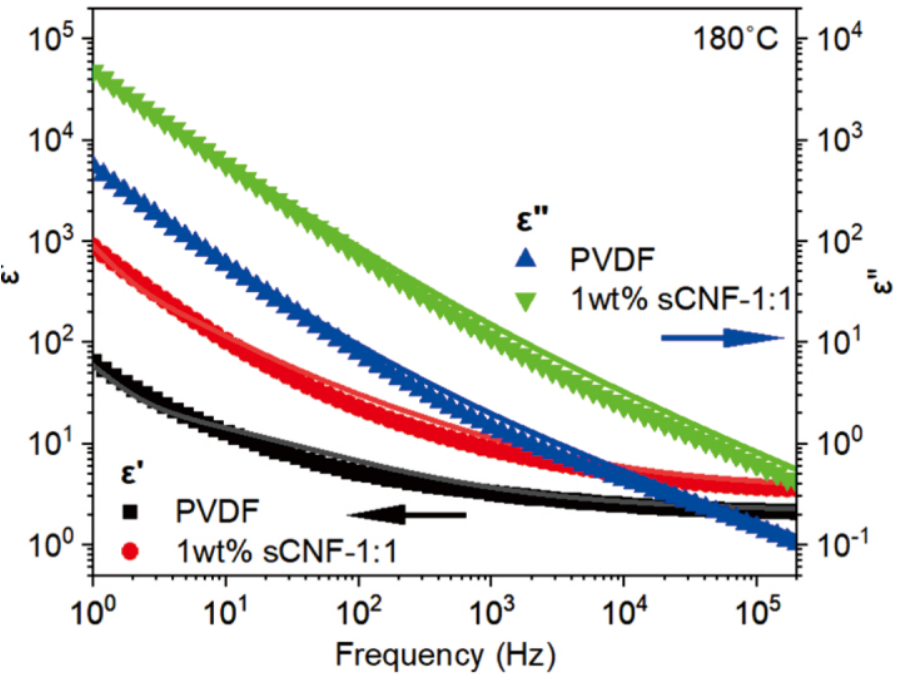

Fig. 10 Comparison between experimental data and $\mathrm{HN}$ fitting results of real and imaginary parts of dielectric permittivity at $180{ }^{\circ} \mathrm{C}$. Symbols: experimental data; solid lines: $\mathrm{HN}$ fitting results.

asymmetric broadenings of the relaxations.

Moreover, introducing CNFs to PVDF matrix reduced both $\Delta \varepsilon_{1}$ and $\Delta \varepsilon_{2}$, compared with pure PVDF (Table 3 and Fig.11). It is worthy to mention here that conductive relaxation experienced more significant reduction, compared with MWS relaxation. According to the discussion in Section 3.2.1, in the PVDF/CNF nanocomposites, the charge carriers from both PVDF and untreated CNFs were largely confined in the vicinity of CNF aggregates, related to MWS polarization, which should be responsible for such a dramatic reduction, contributing to the reduced electrical conductivity at low and medium frequencies in PVDF/CNF nanocomposites. Via 2S-SPI modification, $\Delta \varepsilon_{1}$ greatly increased, indicating the remarkable enhancement of conductivity relaxation at both $180{ }^{\circ} \mathrm{C}$ and $160{ }^{\circ} \mathrm{C}$. The reduction of $\Delta \varepsilon_{2}$ likely resulted from the varied MWS mechanism in the nanocomposites, compared with pure PVDF. $\Delta \varepsilon_{2}$ was also recovered by 2S-SPI modification to different levels, in comparison with PVDF/CNF nanocomposites. The greater amount of enhancement of $\Delta \varepsilon_{1}$ of $\mathrm{PVDF} / \mathrm{sCNF}$ nanocomposites, as

Table 3. Havriliak-Negami function parameters for conductivity and MWS relaxations at $180{ }^{\circ} \mathrm{C}$.

\begin{tabular}{|c|c|c|c|c|c|c|c|c|}
\hline \multirow{2}{*}{\multicolumn{2}{|c|}{ Relaxation type }} & \multirow{2}{*}{ PVDF } & \multirow{2}{*}{$\begin{array}{c}0.1 \mathrm{wt} \% \\
\mathrm{CNF}\end{array}$} & \multirow{2}{*}{$\begin{array}{l}0.1 \mathrm{wt} \% \\
\text { sCNF-1:1 }\end{array}$} & \multirow{2}{*}{$\begin{array}{l}0.1 \mathrm{wt} \% \\
\text { sCNF-1:3 }\end{array}$} & \multirow{2}{*}{$\begin{array}{l}1 \mathrm{wt} \% \\
\mathrm{CNF}\end{array}$} & \multirow{2}{*}{$\begin{array}{c}1 \mathrm{wt} \% \\
\text { sCNF-1:1 }\end{array}$} & \multirow{2}{*}{$\begin{array}{c}1 \mathrm{wt} \% \\
\text { sCNF-1:3 }\end{array}$} \\
\hline & & & & & & & & \\
\hline & $\alpha_{1}$ & 1.00 & 1.00 & 1.00 & 1.00 & 1.00 & 0.92 & 0.93 \\
\hline Conductivity & $\beta_{1}$ & 1.00 & 1.00 & 1.00 & 0.94 & 0.94 & 0.94 & 0.95 \\
\hline \multirow[t]{3}{*}{ relaxation } & $\tau_{1}$ & $2.81 \mathrm{E}-01$ & $1.21 \mathrm{E}-01$ & $1.05 \mathrm{E}-01$ & $2.03 \mathrm{E}-01$ & $1.65 \mathrm{E}-01$ & $3.77 \mathrm{E}-02$ & $2.21 \mathrm{E}-02$ \\
\hline & $\Delta \varepsilon_{1}$ & 180.75 & 16.73 & 1094.49 & 749.26 & 7.50 & 2828.70 & 1940.00 \\
\hline & $\alpha_{2}$ & 1.00 & 0.54 & 1.00 & 0.58 & 1.00 & 0.60 & 0.67 \\
\hline \multirow{3}{*}{ MWS } & $\beta_{2}$ & 0.45 & 1.00 & 0.46 & 1.00 & 0.25 & 1.00 & 0.97 \\
\hline & $\tau_{2}$ & $1.66 \mathrm{E}-02$ & $3.77 \mathrm{E}-05$ & $6.02 \mathrm{E}-03$ & $3.24 \mathrm{E}-05$ & $3.05 \mathrm{E}-03$ & $3.47 \mathrm{E}-05$ & $2.10 \mathrm{E}-05$ \\
\hline & $\Delta \varepsilon_{2}$ & 19.30 & 3.24 & 14.94 & 19.25 & 1.29 & 3.41 & 3.78 \\
\hline
\end{tabular}



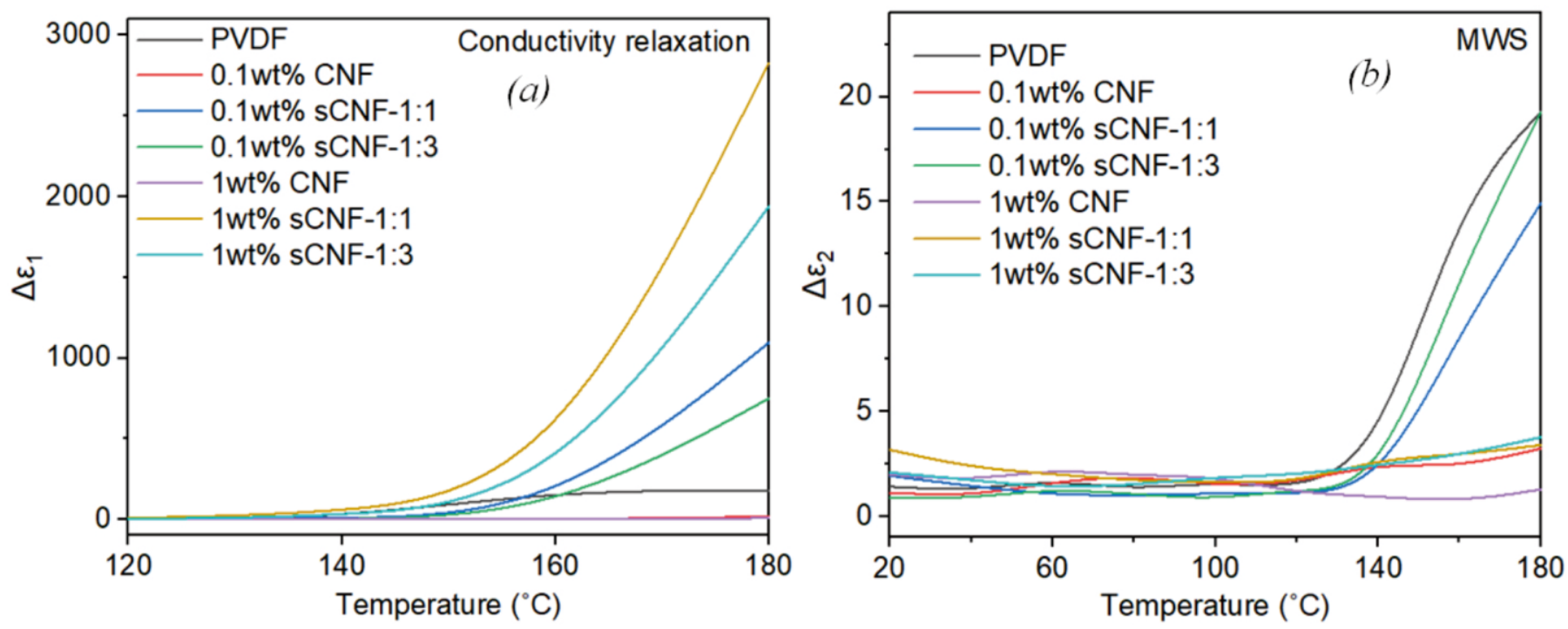

Fig. 11 Dielectric relaxation strengths of (a) conductivity relaxation, (b) MWS relaxation of pure PVDF and the nanocomposites as a function of temperature.

temperature increased, revealed the efficient synergy between $2 \mathrm{~S}$ SPI modification and high temperature on conductivity relaxation, compared with both pure PVDF and PVDF/CNF nanocomposites. Oppositely, the relaxation strengths of both relaxations barely varied in PVDF/CNF nanocomposites, as temperature increased, i.e. weak temperature dependence, and this is consistent with the lower activation energies of both relaxation mechanisms in $\mathrm{PVDF} / \mathrm{CNF}$ nanocomposites.

In summary, 2S-SPI modification was able to enhance both conductivity relaxation and MWS relaxation, with remarkable enhancement observed in conductivity relaxation, regardless of the content of CNFs and the amount of 2S-SPI surfactant. The surface modification also led to greater temperature sensitivity of both types of relaxations in the sCNF nanocomposites, compared with $\mathrm{CNF}$ nanocomposites with pristine CNFs.

\subsubsection{Discussion on the Effects of Dielectric Relaxation on Electrical Conduction of the Nanocomposites}

As discussed above, both conductivity relaxation and MWS relaxation had distinctive impact on electrical conduction. In PVDF/CNF nanocomposites, the MWS relaxation had prominent effects on the electrical conduction at low and medium frequencies. The short-range charge hopping near the interfaces was active, associated with a large number of confined charge carriers. However, due to poor dispersion and low CNF concentration, the lack of effective conductive pathways suppressed the conductivity relaxation. Due to the decoupling between MWS relaxation and conductivity relaxation, the contribution of MWS to electrical conduction stands out in PVDF/CNF nanocomposites.

In PVDF/sCNF nanocomposites, although the dispersion was greatly improved, due to low CNFs concentration and high energy barrier of 2S-SPI layer, both short-range and long-range charge hopping were suppressed. Therefore, at low temperatures, the conductivity relaxation was weak. As temperature increased, the charge carriers were successfully thermally activated to overcome the energy barrier, and conductivity relaxation was greatly enhanced. With the help of uniform CNF dispersion, the transition from short- range to long-range hopping was favored. In this case, the contribution of MWS polarization to electrical conduction was substantially weakened, although the MWS polarization was also improved by 2S-SPI modification. Two reasons are likely responsible for this weakened contribution: very strong conductivity relaxation, and coupling between conductivity relaxation and MWS relaxation. The comparison between pure PVDF and PVDF/sCNF nanocomposites in both (similar) electric conduction and (largely different) conductivity relaxation at high temperatures, suggested that the enhancement of conductivity relaxations was majorly contributed by the charge carriers from $\mathrm{CNFs}$ in $\mathrm{PVDF} / \mathrm{sCNF}$ nanocomposites. From the remarkably reduced electrical conductivity in PVDF/CNF nanocomposites, it was believed that charge carriers from PVDF polymer were largely confined around CNFs aggregates, because of the prominent effects of MWS relaxation in PVDF/CNF nanocomposites.

\section{Conclusions}

In this study, 2S-SPI was found to be an effective surfactant to modify carbon nanofibers (CNFs) for polymer nanocomposites. Surface modification of CNFs by 2S-SPI greatly improved dispersion quality of SCNFs in PVDF. The study also revealed the distinctive roles of 2S-SPI surfactant in both electrical and dielectric properties of the nanocomposites, with enhanced activation energy of both electrical conduction and dielectric relaxation found in $2 \mathrm{~S}$ SPI modified nanocomposites. 2S-SPI modification showed significant effects on both MWS relaxation and conductivity relaxation, particularly, at high temperatures. The relaxations strengths of both relaxation processes increased at high temperatures. Moreover, the enhancement of conductivity relaxation was much more sensitive to 2S-SPI modification, compared with MWS relaxation, leading to much stronger relaxation strength of conductivity relaxation. In contrast to the decoupled conductivity relaxation and MWS relaxation in PVDF/CNF nanocomposites, the coupling of the two relaxation mechanisms was achieved by 2S-SPI modification, which further affected the electrical conduction of the nanocomposites. 
2S-SPI surfactant altered the conduction mechanisms of AC conductivity at high temperatures. Without surface modification, the electrical conduction of PVDF/CNF nanocomposites was largely affected by MWS relaxation, while the long-range charge hopping was suppressed, leading to reduced electrical conductivity, compared with pure PVDF. After 2S-SPI modification, the long-range charge hopping became dominant factor of the electrical conduction, leading to recovered electrical conductivity, although the strength of MWS relaxation was also enhanced by 2 S-SPI surfactant.

\section{Conflict of interest}

There are no conflicts to declare.

\section{Acknowledgements}

This work is supported by the USDA National Institute of Food and Agriculture, AFRI project [2017-67021-26604]. The author is also grateful to Archer Daniels Midland (ADM) for generously providing SPI.

\section{Reference}

1. Y. Jewel, T. Liu, A. Eyler, W. Zhong and J. Liu, J. Phys. Chem. C, 2015, 119, 26760-26767.

2. A. Ortiz-Acevedo, H. Xie, V. Zorbas, W. Sampson, A. Dalton, R. Baughman, R. Draper, I. Musselman and G. Dieckmann, J. Am. Chem. Soc., 2005, 127, 9512-9517.

3. S. Karajanagi, H. Yang, P. Asuri, E. Sellitto, J. Dordick and R. Kane, Langmuir, 2006, 22, 1392-1395.

4. F. Song, D. Tang, X. Wang and Y. Wang, Biomacromolecules, 2011, 12, 3369-3380.

5. J. Ji, B. Lively and W. Zhong, Mater Express, 2012, 2, 76-82.

6. S. Tay, S. Kasapis, C. Perera and P. Barlow, J. Agric. Food Chem., 2006, 54, 6046-6053.

7. A. Velarde-Salcedo, A. Barrera-Pacheco, S. Lara-González, G. Montero-Morán, A. Díaz-Gois, E. de Mejia and A. de la Rosa, Food chemistry, 2013, 136, 758-764.

8. P. Tomar, K. Nikhil, A. Singh, P. Selvakumar, P. Roy and A. Sharma, Biochem. Biophys. Res. Commun., 2014, 448, 349-354.

9. D. Sung, K. Ahn, S. Lim and S. Oh, J. Sci. Food Agric., 2014, 94, 2482-2487.

10. J. Nordlee, S. Taylor, J. Townsend, L. Thomas and R. Bush, New Engl. J. Med., 1996, 334, 688-692.

11. S. Saptarshi, A. Duschl and A. Lopata, J. Nanobiotechnology, 2013, 11, DOI: 10.1186/1477-3155-11-26.

12. R. Haggenmueller, H. Gommans, A. Rinzler, J. Fischer and K. Winey, Chem. Phys. Lett., 2000, 330, 219-225.

13. C. Park, Z. Ounaies, K. Watson, R. Crooks, J. Smith, S. Lowther, J. Connell, E.. Siochi, J. Harrison and T. Clair, Chem. Phys. Lett., 2002, 364, 303-308.

14. F. Du, J.. Fischer and K. Winey, J. Polym. Sci., Part B: Polym. Phys., 2003, 41, 3333-3338.

15. Y. Geng, M. Liu, J. Li, X. Shi and J. Kim, Composites Part A, 2008, 39, 1876-1883.

16. N. Grossiord, J. Loos, O. Regev and C. Koning, Chem. Mater., 2006, 18, 1089-1099.

17. X. Gong, J. Liu, S. Baskaran, R. Voise and J. Young, Chem. Mater., 2000, 12, 1049-1052.

18. L. Vaisman, G. Marom and H. Wagner, Adv. Funct. Mater., 2006,
16, 357-363.

19. J. Sung, H. Kim, H. Jin, H. Choi and I. Chin, Macromolecules, 2004, 37, 9899-9902.

20. M. Strano, V. Moore, M. Miller, M. Allen, E. Haroz, C. Kittrell, R. Hauge and R. Smalley, J. Nanosci. Nanotechno., 2003, 3, 8186.

21. J. Yu, N. Grossiord, C. Koning and J. Loos, Carbon, 2007, 45, 618-623.

22. E. Whitsitt, A. Barron, Nano Lett., 2003, 3, 775-778.

23. T. Kim, C. Doe, S. Kline and S. Choi, Adv. Mater., 2007, 19, 929-933.

24. A. Maestro, E. Guzmán, E. Santini, F. Ravera, L. Liggieri, F. Ortega and R. Rubio, Soft Matter, 2012, 8, 837-843.

25. M. Osman, J. Rupp and U. Suter, Polymer, 2005, 46, 8202-8209.

26. P. Jash, C. Wilkie, Polym. Degradation Stab., 2005, 88, 401-406.

27. A. Uygun, O. Turkoglu, S. Sen, E. Ersoy, A. Yavuz and G. Batir, Curr. Appl. Phys., 2009, 9, 866-871.

28. S. El-Sayed, Physica. B ., 2014, 454, 197-203.

29. C. Chanmal, J. Jog, Express Polym. Lett., 2008, 2, 294-301.

30. Z. Zheng, B. Li, J. Appl. Polym. Sci., 2018, 135, 46882.

31. A. Sulong, N. Muhamad, J. Sahari, R. Ramli, B. Deros and J. Park, Eur. J. Sci. Res., 2009, 29, 13-21.

32. J.. Hong, L. Schadler, R. Siegel and E. Martensson, J. Mater Sci., 2006, 41, 5810-5814.

33. K. Funke, Prog. Solid State Chem., 1993, 22, 111-195.

34. M. Mostafa,A. Hassen, Phase Transitions, 2006, 79, 305-321.

35. X. Jiang, X. Zhao, G. Peng, W. Liu, K. Liu and Z. Zhan, Curr. Appl. Phys., 2017, 17, 15-23.

36. S. Dash, Ph.D. dissertation, Shiksha o Anusandhan University, 2014.

37. H. Lu, X. Zhang, J. Appl. Phys., 2006, 100, 054104.

38. M. Hernández, J. Carretero-González, R. Verdejo, T. Ezquerra and M. López-Manchado, Macromolecules, 2009, 43, 643-651.

39. Y. Feng, W. Li, Y. Hou, Y. Yu, W. Cao, T. Zhang and W. Fei, J. Mater. Chem., 2015, 3, 1250-1260.

40. B. Money, K. Hariharan and J. Swenson, Solid State Ionics, 2014, 262, 785-789.

41. B. Roling, A. Happe, K. Funke and M. Ingram, Phys. Rev. Lett., 1997, 78, 2160-2163.

42. G. Pike, Phys. Rev. B: Condens. Matter, 1972, 6, 1572

43. D. Tee, M. Mariatti, A. Azizan, C. See and K.. Chong, Compos. Sci. Technol., 2007, 67, 2584-2591.

44. D. Sinirlioglu, A. Muftuoglu, J. Polym. Res., 2015, 22, 1-16.

45. T. Satyavani, B. Kiran, V. Kumar, A. Kumar and S. Naidu, Eng. Sci. Technol. Int J., 2016, 19, 40-44.

46. J. Mijovic, H. Lee, J. Kenny and J. Mays, Macromolecules, 2006, 39, 2172-2182.

47. R. Gregorio Jr, M. Cestari, J. Polym. Sci., Part B: Polym. Phys., 1994, 32, 859-870.

48. A. Linares, A. Nogales, D. Rueda and T. Ezquerra, J. Polym. Sci., Part B: Polym. Phys., 2007, 45, 1653-1661.

49. E. Neagu, J. Hornsby and D. Das-Gupta, J. Phys. D: Appl. Phys., 2002, 35, 1229.

50. H. Rekik, Z. Ghallabi, I. Royaud, M. Arous, G. Seytre, G. Boiteux and A. Kallel, Composites Part B, 2013, 45, 1199-1206.

51. P. Steeman, F. Maurer and J. Van Turnhout, Polym. Eng. Sci., 1994, 34, 697-706.

52. M. Arous, I. Amor, A. Kallel, Z. Fakhfakh and G. Perrier, $J$ 
Phys. Chem. Solids, 2007, 68, 1405-1414.

53. B. Lu, K. Lamnawar, A. Maazouz and H. Zhang, Soft Matter, 2016, 12, 3252-3264.

54. S. Lanceros-Mendez, J. Mano, A. Costa and V. Schmidt, J. Macromol. Sci. B, 2001, 40, 517-527.
55. J. Mijovic, J. W. Sy and T. K. Kwei, Macromolecules, 1997, 30, 3042-3050.

56. A. Ghosh, A. Naskar, D. Khastgir and S. K. De, Polymer, 2001, 42, 9849-9853.

57. D. Boese, F. Kremer, Macromolecules, 1990, 23, 829-835. 